\title{
Asymmetric optical loop mirror: analysis of an all-optical switch
}

\author{
Michael G. Kane, Ivan Glesk, Jason P. Sokoloff, and Paul R. Prucnal
}

\begin{abstract}
We present an analysis of the optical loop mirror in which a nonlinear optical element is asymmetrically placed in the loop. This analysis provides a general framework for the operation of a recently invented ultrafast all-optical switch known as the terahertz optical asymmetric demultiplexer. We show that a loop with small asymmetry, such as that used in the terahertz optical asymmetric demultiplexer, permits low-power ultrafast all-optical sampling and demultiplexing to be performed with a relatively slow optical nonlinearity. The size of the loop is completely irrelevant to switch operation as long as the required degree of asymmetry is accommodated. This is therefore the first low-power ultrafast all-optical switch that can be integrated on a single substrate.
\end{abstract}

Key words: Optical loop mirror, semiconductor optical amplifier, optical switching, optical demultiplexing.

\begin{abstract}
Introduction
Various all-optical switches have been proposed and demonstrated. All of these switches use an optical nonlinearity of some type, since all-optical switching requires a nonlinear function to be performed on one or more optical fields. The optical nonlinearities are produced by electronic excitations of a material, usually a semiconductor or a glass. Nonlinearities tend to be relatively strong and slow when the frequency of an optical field resonates with the frequency of a long-lived excitation, permitting slow switching with small switching energy. An example of such a nonlinearity is modification of the refractive index in a semiconductor through the photogeneration of electron-hole pairs. On the other hand, excitations produced when the frequency of an optical field is detuned from the frequencies of excitation or when the excitations are short lived lead to relatively weak, fast nonlinearities. These permit fast switching but require large switching energy. An example
\end{abstract}

M. G. Kane is with Siemens Corporate Research, Inc., 755 College Road East, Princeton, New Jersey 08540; he is also with the Department of Electrical Engineering, Princeton University, Princeton, New Jersey 08544. I. Glesk, J. P. Sokoloff, and P. R. Prucnal are with the Center for Photonics and Optoelectronic Materials, Department of Electrical Engineering, Princeton University, Princeton, New Jersey 08544.

Received 16 June 1993; revised manuscript received 22 April 1994.

0003-6935/93/296833-10\$06.00/0.

(C) 1994 Optical Society of America. of this type of excitation is the optical Kerr effect in optical fiber, arising from nonresonant excitation of bound electrons in the silica glass.

The fast response time of the Kerr nonlinearity in silica fiber has prompted the development of several types of all-fiber switches, capable of switching pulses that are a picosecond long or less. Because the optical Kerr effect in silica fiber is weak, these devices require large switching energies or long lengths of fiber to extend the interaction distance, or both. ${ }^{1-4}$

In order to reduce the size and the power requirements of an all-optical switch, researchers have investigated other materials with stronger nonlinearities. ${ }^{5-7}$ In particular, switches have been constructed by incorporation of a nonlinear element (NLE) in a loop mirror, permitting the switch to take advantage of the loop's highly stable zero-background output. . $^{8}$ However, in most switches of this type the basic speed-power trade-off of the nonlinear element remains. For example, Eiselt demonstrated a switch with a semiconductor optical amplifier in a fiber loop; the amplifier's strong, slow nonlinearity permits the switch to operate with a switching energy of only 0.4 pJ, but the time resolution of the switch is limited by the amplifier's 400-ps gain recovery time. ${ }^{12}$

In contrast, a terahertz optical asymmetric demultiplexer (TOAD) has been demonstrated that directly addresses the problem created by the speed-power trade-off by permitting a relatively slow, sensitive nonlinearity to perform ultrafast switching. ${ }^{13}$ By placing a NLE in a short loop mirror with slight asymmetry, one can reduce the time resolution to far 
less than the recovery time of the optical nonlinearity. Using semiconductor optical amplifiers with a gain recovery time of $\sim 1 \mathrm{~ns}$, researchers have used this architecture to perform optical time-division demultiplexing at $9 \mathrm{Gbit} / \mathrm{s}$ (Ref. 14) and $50 \mathrm{Gbit} / \mathrm{s}^{15}$ Recently demultiplexing was demonstrated at 250 Gbit/s with a bit-error rate of less than $10^{-9}$ and a switching energy of $800 \mathrm{fJ} .{ }^{16}$

Our purpose in this paper is to provide a general analytical framework for the operation of the TOAD switch and to place it within the broader context of other switches that use a nonlinear optical element in a loop mirror. After the analysis, we provide experimental data to illustrate the main results and describe some applications.

\section{Analysis of Switch Operation}

\section{General Formulation}

A linear optical loop mirror consists of a $2 \times 23-\mathrm{dB}$ coupler with two ports joined. Its properties and applications are described in Refs. 17-20. Here we consider a loop mirror in which a NLE is placed asymmetrically in the loop (Fig. 1). The degree of asymmetry is represented by the distance $\Delta x$ between the NLE and the midpoint of the loop. The control is exercised by applying an optical control pulse to the NLE, altering its absorption and/or refractive index, and switching some portion of the input signal to the output for the duration of the sampling window created by the control pulse. The optical input enters the loop through port 1 of the coupler, and the switched optical output exits from port 2.

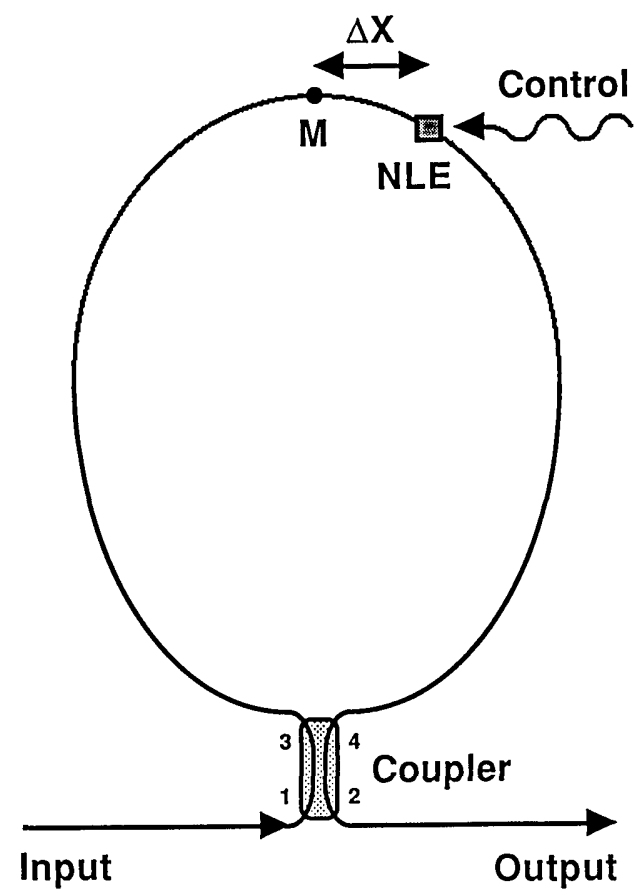

Fig. 1. Optical loop mirror with a nonlinear element (NLE) placed asymmetrically in the loop at a distance $\Delta x$ from the loop's midpoint M. The optical control modifies the optical properties of the NLE, causing the input signal to be switched to the output.
In the absence of a control signal the switch operates as a linear loop mirror. The input signal enters port 1 of the coupler with an intensity $I$, then splits into two fields of intensity $I / 2$ at ports 3 and 4, one propagating clockwise (CW) in the loop and the other counterclockwise (CCW). (CW does not stand for continuous wave in what follows.) As it crosses from one side of the coupler to the other, the CCW field undergoes a $\pi / 2$ phase delay relative to the $\mathrm{CW}$ field. Then both fields travel the same distance in the loop. To reach the output at port 2, the CCW field must cross the coupler again. As a result, at the output there is a superposition of two fields having equal amplitudes but a $\pi$ phase difference. The resulting destructive interference prevents optical power from exiting from the output, provided that the relative polarization of the two fields does not change as they traverse the loop. Therefore all of the optical power entering the input must be reflected. (In actual use an optical isolator can be placed at the input to absorb the reflected power.) The loop can thus be regarded as a white-light interferometer in which the two arms are distinguished by the two directions of propagation.

If a constant control signal is applied to the NLE, its optical characteristics will be modified. However, the two counterpropagating modes will interfere in the same way as in the absence of the control signal because the counterpropagating fields still experience identical propagation characteristics. But if a timevarying control signal is applied to the NLE, the optical properties of the NLE become time dependent. As a result, the destructive interference that leads to field cancellation at the output is disturbed. If a portion of the light traveling in the loop experiences an absorption or an index difference relative to its counterpropagating complement, light will emerge from the output of the coupler.

Now we analyze the time-dependent operation of the loop in more detail. In our analysis we make several assumptions:

(1) Optical couplers are ideal. Commercially available couplers approximate this ideal adequately.

(2) The input signal's intensity is sufficiently small that it does not modify the optical properties of the NLE, and neither the input nor the control signal induces nonlinearities in the other parts of the loop.

(3) The relative polarization of the counterpropagating $\mathrm{CW}$ and $\mathrm{CCW}$ fields is maintained as they traverse the loop.

(4) No reflections occur at the interface between the NLE and the loop medium. This assumption is easily approximated in practice by use of antireflection coatings.

(5) The input signal can be distinguished from the control signal. One realizes this in practice by keeping the control signal out of the loop or by using suitable wavelength or polarization filters. Similarly we ignore noise that may be generated by the 
NLE, since it can largely be blocked with suitable output filters.

The total field at port 2 of the loop is the sum of two components, one from the $\mathrm{CW}$ field and one from the CCW field. The output intensity is

$$
I_{\text {out }}(t)=\frac{c n}{8 \pi}\left|E_{\mathrm{CW}}(t)+E_{\mathrm{CCW}}(t)\right|^{2},
$$

where $n$ is the index of the medium and $E_{\mathrm{CW}(\mathrm{CCW})}(t)$ represents the complex amplitude of the CW (CCW) field as it exits from port 2. For a monochromatic continuous-wave optical input of frequency $\omega$, the amplitudes at port 2 are

$$
\begin{aligned}
E_{\mathrm{CW}}(t) & =\left(\frac{2 \pi I_{\mathrm{in}}}{c n}\right)^{1 / 2} \exp \left[-i \omega t+i \phi_{\mathrm{CW}}(t)-\gamma_{\mathrm{CW}}(t)\right], \quad(2) \\
E_{\mathrm{CCW}}(t) & =-\left(\frac{2 \pi I_{\mathrm{in}}}{c n}\right)^{1 / 2} \exp \left[-i \omega t+i \phi_{\mathrm{CCW}}(t)-\gamma_{\mathrm{CCW}}(t)\right],
\end{aligned}
$$

where $I_{\text {in }}$ is the input intensity incident upon port 1 and $\phi_{\mathrm{CW}(\mathrm{CCW})}(t)$ and $\gamma_{\mathrm{CW}(\mathrm{CCW})}(t)$ represent the timedependent phase shift and attenuation that the $C W$ (CCW) field emerging from port 2 at time $t$ experienced as it traversed the NLE. The phase shift and attenuation can depend on the frequency $\omega$. The expression for $E_{\mathrm{CCW}}(t)$ has a negative sign to account for the two $\pi / 2$ phase shifts that the counterclockwise field experiences crossing the coupler twice.

The attenuation and phase responses of the NLE to the control signal can be complicated functions of time. These responses represent changes in absorption and refractive index, and they depend on the control signal and the properties of the NLE. These details are not of interest here, although we do make some observations about certain types of response characteristics later in the paper.

Using exponential notation would be burdensome for our purposes, so we represent attenuation without it, defining the NLE's transmission $A(t)$ for the clockwise and counterclockwise fields as

$$
A_{\mathrm{CW}(\mathrm{CCW})}(\mathrm{t}) \triangleq \exp \left[-\gamma_{\mathrm{CW}(\mathrm{CCW})}(t)\right] \text {. }
$$

We define the sampling function $S(t)$ as the timedependent output intensity $I_{\text {out }}(t)$ divided by the continuous-wave input intensity $I_{\text {in }}$. Then, combining Eqs. (1)-(4), we get

$$
\begin{aligned}
S(t)= & 1 / 4\left\{A_{\mathrm{CW}}^{2}(t)+A_{\mathrm{CCW}}^{2}(t)-2 A_{\mathrm{CW}}(\mathrm{t}) \mathrm{A}_{\mathrm{CCW}}(t)\right. \\
& \left.\times \cos \left[\phi_{\mathrm{CW}}(t)-\phi_{\mathrm{CCW}}(t)\right]\right\} .
\end{aligned}
$$

This is the general expression describing the timedependent sampling of the loop's optical input by its output.

Often an NLE is used in a manner in which the time variation of either the transmission or the phase shift is dominant. In these cases, we can simplify
Eq. (5), either by approximating $\phi_{\mathrm{CW}}(t)$ and $\phi_{\mathrm{CCW}}(t)$ as a constant phase shift $\phi_{0}$ or by approximating $A_{\mathrm{CW}}(t)$ and $A_{\mathrm{CCW}}(t)$ as a constant transmission $A_{0}$. We refer to these as amplitude-modulated and phase-modulated operation, respectively.

\section{Zero-Length Approximation}

We now employ an approximation that we call the zero-length approximation. The condition for validity of this approximation is as follows. We define the loop's time asymmetry $\Delta t \triangleq \Delta x / v_{\text {loop }}$, where $v_{\text {loop }}$ is the speed of light in the loop medium, and similarly we define the transit time of the NLE as $T_{\text {transit }} \triangleq L / v_{\text {NLE }}$, where $L$ is the length of the NLE and $v_{\mathrm{NLE}}$ is the speed of light in the NLE. The zero-length approximation applies if $T_{\text {transit }} \ll \Delta t$. This approximation leads to a simpler analysis, but later in the paper we remove it.

In the zero-length approximation we therefore ignore the finite length of the NLE and treat it as an asymmetrically placed point element. Then the amplitude (and phase) changes experienced by the two fields are identical except for a time shift, and we can introduce new transmission and phase-shift variables without the CW and CCW subscripts:

$$
\begin{aligned}
& A_{\mathrm{CW}}(t-\Delta t)=\mathrm{A}_{\mathrm{CCW}}(t+\Delta t) \triangleq A(t), \\
& \phi_{\mathrm{CW}}(t-\Delta t)=\phi_{\mathrm{CCW}}(t+\Delta t) \triangleq \phi(t) .
\end{aligned}
$$

Substituting Eqs. (6) and (7) into Eq. (5), we get

$$
\begin{aligned}
S(t)= & 1 / 4\left\{A^{2}(t+\Delta t)+A^{2}(t-\Delta t)-2 A(t+\Delta t) A(t-\Delta t)\right. \\
& \times \cos [\phi(t+\Delta t)-\phi(t-\Delta t)]\} .
\end{aligned}
$$

\section{Loop with Large Asymmetry}

It is useful to analyze two special loop configurations. The first is a loop with large asymmetry. Representatives of this type of switch can be found in the research described in Refs. 9-12. To define this configuration, we consider the control signal to be a pulse of some shape. The NLE responds to this pulse. Before the control pulse appears, the transmission $A(t)$ and the phase shift $\phi(t)$ of the NLE have their steady-state values $A_{0}$ and $\phi_{0}$, respectively, and after some finite time $T_{\text {control }}$, they return to these values. We define a zero-background transmission $\tilde{A}(t)$ and phase shift $\tilde{\phi}(t)$ such that

$$
\begin{gathered}
\tilde{A}(t) \triangleq A(t)-A_{0}, \\
\tilde{\phi}(t) \triangleq \phi(t)-\phi_{0} .
\end{gathered}
$$

Thus these two new variables are zero outside some period of duration $T_{\text {control}}$.

A loop with large asymmetry is one in which the inequality $2 \Delta t>T_{\text {control }}$ is satisfied. Continuing to use the zero-length approximation for now, we can see that the period of time that $\tilde{A}(t+\Delta t)$ and $\tilde{\phi}(t+\Delta t)$ are nonzero does not overlap the period of time that $\tilde{A}(t-\Delta t)$ and $\tilde{\phi}(t-\Delta t)$ are nonzero. (We assume that successive control pulses are separated 
by more than $2 \Delta t+T_{\text {control. }}$ ) Applying these conditions to Eq. (8), we get

$$
\begin{aligned}
S(t)= & \frac{\tilde{A}^{2}(t+\Delta t)}{4}+A_{0} A(t+\Delta t) \sin ^{2} \frac{\tilde{\phi}(t+\Delta t)}{2} \\
& +\frac{\tilde{A}^{2}(t-\Delta t)}{4}+A_{0} A(t-\Delta t) \sin ^{2} \frac{\tilde{\phi}(t-\Delta t)}{2} .
\end{aligned}
$$

The first two terms on the right-hand side are identical to the second two terms, apart from a time

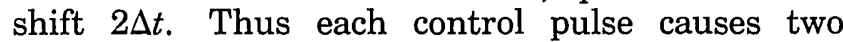
nonoverlapping sampling windows to be opened between input and output. The two windows are identical but separated in time by $2 \Delta t$. The duration of each window is $T_{\text {control }}$, and therefore switching cannot be performed faster than the recovery time of the NLE. As a result, in this configuration the basic speed-power trade-off of the NLE remains.

\section{Loop with Small Asymmetry}

Now we consider the second special loop configuration, representing the configuration used in the TOAD switch. ${ }^{13}$ In some ways it is the opposite of the loop with large asymmetry just described. Now the loop's time asymmetry is much shorter than the response of the NLE to the control pulse; thus the inequality $2 \Delta t \ll T_{\text {control }}$ is satisfied. In general this yields rather complicated results unless we specify that the response of the NLE's optical properties to the control pulse consists of a fast transition with a short rise time, followed by a period of slow relaxation with a long fall time, with $\tau_{\text {rise }}<\Delta t \ll \tau_{\text {fall }}$. Therefore when we call this a loop with small asymmetry, it is with the understanding that the small asymmetry is combined with occasional rapid transitions in the transmission and/or the phase shift.

To analyze this configuration, we apply the approximations $\tau_{\text {rise }}=0$ and $\tau_{\text {fall }}=\infty$. Thus at some time $t_{0}$ the transmission $A(t)$ steps from a value of $A_{0}$ to $A_{1}$, or the phase shift $\phi(t)$ steps from a value of $\phi_{0}$ to $\phi_{1}$, or both. After the step, variations in $A(t)$ and $\phi(t)$ are neglected until the next step. By inspection of Eq. (8) we can determine the response of the sampling function to the step. Before $t=t_{0}-\Delta t$ and after $t=t_{0}+\Delta t$, the sampling function $S(t)$ is approximately zero. The first two terms in the braces are approximately canceled by the third term because of the slow variation in the transmission and the phase shift. But for times $t_{0}-\Delta t<t<t_{0}+\Delta t$ the cancelation does not occur, and the sampling function has a constant level:

$$
S(t)=\left\{\begin{array}{cl}
1 / 4\left[A_{0}^{2}+A_{1}^{2}-2 A_{0} A_{1} \cos \left(\phi_{0}-\phi_{1}\right)\right] \\
\quad \text { for } t_{0}-\Delta t<t<t_{0}+\Delta t \\
0 \quad \text { otherwise }
\end{array} .\right.
$$

Thus the loop with small asymmetry permits a rectangular sampling window to be created from a single rapid transition, with the width of the sam. pling window determined by the time asymmetry of the loop. This explains why the TOAD switch can achieve a time resolution far below the recovery time of the optical nonlinearity. Furthermore, the size of the loop is completely irrelevant to switch operation as long as there is enough room for the required asymmetry.

The only restriction on operation is that another sampling window cannot be created until a time greater than $\tau_{\text {fall }}$ has elapsed, permitting the nonlinearity to recover. But modeling the transmission and the phase shift as a step function involves two approximations whose validity must be addressed.

First, in reality, $\tau_{\text {rise }}>0$. As a result, the rising and falling transitions of the rectangular pulse described by Eq. (12) are not instantaneous, but require a time $\tau_{\text {rise }}$. This transition time can be quite small, even for slow nonlinearities such as those associated with interband transitions in semiconductors. The reason is as follows: When we speak of an optical nonlinearity in a material as fast or slow, we are characterizing the time required for the optical properties to return to their normal, linear values after the incident optical power is removed. The time required for the nonlinearity to turn on can be much shorter than this, since in the presence of an incident optical field the electronic excitations can be induced rapidly. As a first approximation, the time required for an excited state to be generated is shorter than its decay time by a factor equal to the average photon occupation number of all the field modes capable of inducing the excitation. The large occupation numbers achievable with laser excitation permit slow nonlinearities to turn on rapidly. For example, gain nonlinearities associated with interband transitions in semiconductor optical amplifiers can exhibit subpicosecond turn-on times. ${ }^{21,22}$

Second, $\tau_{\text {fall }}$ is not infinite. As a result, the top of the rectangular pulse described by Eq. (12) has a descending slope. In addition, after the pulse has ended, a small residual intensity remains at the output for a period $\tau_{\text {fall }}$. However, it is straightforward to show that this error is proportional to $\left(\Delta t / \tau_{\text {fall }}\right)^{2}$ and can be reduced to small values.

\section{Effect of Finite Nonlinear-Element Length}

Up to this point, we have considered the asymmetric loop mirror with the approximation that the NLE has zero length. This is usually a good approximation in a loop with large asymmetry, since the loop's asymmetry is generally much larger than the length of the NLE. But in a loop with small asymmetry, such as the TOAD switch, this approximation leads to an incorrect conclusion. It suggests that the minimum duration of the sampling window is determined ultimately by the rise time of the nonlinearity, since in principle the NLE can be placed arbitrarily close to the center of the loop. In this subsection we show that the finite length $L$ of the NLE leads to an additional limitation: a nonlinear element can produce a sampling window with a total duration no shorter than twice the NLE's transit time, with a full 
width at half-maximum (FWHM) approximately equal to the transit time. If a typical semiconducting material is used as the nonlinear element, this implies that, with a $100-\mu \mathrm{m}$-long NLE, the FWHM of the sampling window is limited by the finite-length effect to $\sim 1 \mathrm{ps}$.

If the NLE has finite length, we must be more specific about the definition of the time asymmetry $\Delta t$. We define it as the propagation delay from the midpoint of the loop to the nearest edge of the NLE. The midpoint of the loop is defined as the point at which half the time required to traverse the loop has elapsed, including the NLE's transit time.

Equation (5) expresses the sampling function in terms of the transmission functions $A_{\mathrm{CW}}(t)$ and $A_{\mathrm{CCW}}(t)$ and the phase shifts $\phi_{\mathrm{CW}}(t)$ and $\phi_{\mathrm{CCW}}(t)$. With the zero-length approximation the two transmission functions are time-shifted replicas of each other, as are the phase shifts [Eqs. (6) and (7)]. In general this is not true for a finite-length NLE. Instead $A_{\mathrm{CW}}(t)$ can have a different shape from $A_{C C W}$ $(t)$, as can $\phi_{\mathrm{CW}}(t)$ from $\phi_{\mathrm{CCW}}(t)$.

First, we analyze amplitude-modulated operation. We must work in terms of the attenuation $\gamma(t)$ instead of the transmission $A(t)$; these are related through Eq. (4). The attenuation $\gamma_{\mathrm{CW}}(t)$ represents the attenuation that the CW field emerging from port 2 of the coupler at time $t$ experienced as it traversed the NLE; the attenuation is similar for the CCW field. To calculate the attenuation, we represent the NLE's attenuation per unit length at a point $x$ in the NLE and a time $t$ as $\alpha(x, t)$, where the origin $x=0$ is at the end of NLE nearest to the loop's midpoint. We perform an integral through the NLE of the attenuation per unit length in the frame of reference of the CW or the CCW fields, which propagate at a speed $v_{\text {NLE. }}$. The two moving frames of reference are treated by the introduction of an additional time integral and a delta function that moves with the appropriate frame of reference:

$$
\begin{aligned}
\gamma_{\mathrm{CW}}(t)= & \int_{-\infty}^{\infty} \mathrm{d} \tau \int_{0}^{L} \mathrm{~d} x \alpha(x, \tau) \\
& \times \delta\left(\tau-t+T_{1 / 2}-\Delta t-x / v_{N L E}\right) \\
\gamma_{\mathrm{CCW}}(t)= & \int_{-\infty}^{\infty} \mathrm{d} \tau \int_{0}^{L} \mathrm{~d} x \alpha(x, \tau) \\
& \times \delta\left(\tau-t+T_{1 / 2}+\Delta t+x / v_{\mathrm{NLE}}\right)
\end{aligned}
$$

Here we have introduced a fixed time delay $T_{1 / 2}$, representing the propagation delay from the loop's midpoint to port 2 of the main coupler.

In order to make the effects of finite NLE length easier to visualize, we apply some simplifying assumptions to Eqs. (13) and (14). The first assumption is that the NLE does not deviate strongly from transparency. Consider the fact that the optical control pulse propagates through the NLE with a fixed speed. Changes in the NLE attenuation $\alpha(x, t)$ are induced by the control pulse. If the NLE is nearly transparent, the control pulse does not change much as it propagates through the NLE, and the NLE attenuation $\alpha(x, t)$ propagates through the NLE without changing shape, much as a pulse traveling at the same speed as the control pulse. In general this transparency assumption leads to results that are correct to first order, and it makes it easier to understand the finite-length effect.

Various methods of introducing the control pulse into the NLE are possible. For example, if it is injected normal to the loop, as suggested by Fig. 1, the entire length of the NLE experiences the effect of the control pulse simultaneously, and $\alpha(x, t)=\alpha(t)$. But here we assume a configuration in which the control pulse is injected into the loop through an intraloop coupler so that it propagates in the loop in a clockwise direction (Fig. 2 ). [If the intraloop coupler does not discriminate between the control and the input signals, using, for example, wavelength or polarization discrimination, it will permit some of the input signal to leave the loop. This loss reduces the sampling function $S(t)$ by a constant factor.] We assume that the control pulse propagates through the NLE at the same speed as the CW and the CCW input fields. This assumption is usually satisfactory, although it may not be if the control and input signals have different polarization states or are widely separated in wavelength. With these assumptions the attenuation in the NLE takes the form of a traveling wave,

$$
\alpha(x, t)=\alpha\left(t-x / v_{\mathrm{NLE}}\right) .
$$

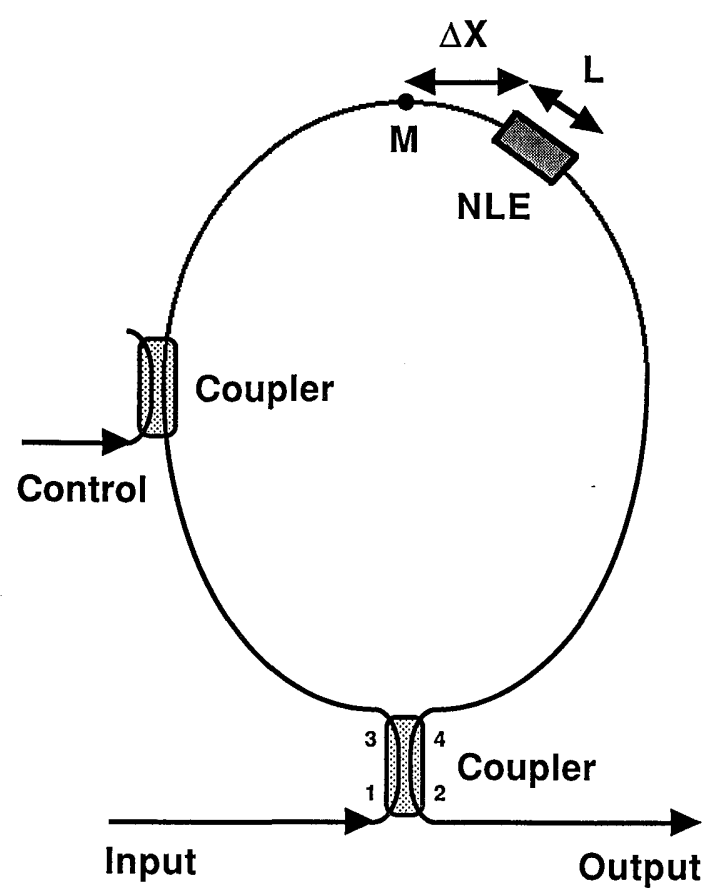

Fig. 2. Optical loop mirror with a finite-length NLE. The optical control is introduced into the loop with an intraloop coupler. 
Substituting Eq. (15) into Eqs. (13) and (14), we get

$$
\begin{aligned}
\gamma_{\mathrm{CW}}(t) & =L \alpha\left(t-T_{1 / 2}+\Delta t\right), \\
\gamma_{\mathrm{CCW}}(t) & =\int_{0}^{L} \mathrm{~d} x \alpha\left(t-T_{1 / 2}-\Delta t-2 x / v_{\mathrm{NLE}}\right) .
\end{aligned}
$$

Because the control pulse and the clockwise input field propagate in the same direction and at the same speed through the NLE, the effect of the control pulse appears to be stationary in the frame of the clockwise input field. This is why the copropagation integral reduces to the simple form of Eq. (16), and the finite length of the NLE has no effect on the shape of $\gamma_{\mathrm{CW}}(t)$. But the counterpropagation integral in Eq. (17) is more complicated because the control pulse and the counterclockwise input field move past each other in the NLE, and the NLE's finite length alters the shape of $\gamma_{\mathrm{CCW}}(t)$.

An analysis of phase-modulated operation follows the same lines. The phase shifts through the NLE $\phi_{\mathrm{CW}}(t)$ and $\phi_{\mathrm{CCW}}(t)$ can be calculated from the spaceand time-dependent phase shift per unit length, which is the NLE's propagation wave number $k(x, t)=\omega n(x, t) / c$, where $n(x, t)$ is the space- and time-dependent refractive index in the NLE. However, in this case, simple propagation integrals such as those in Eqs. (13) and (14) cannot be written because the CW and the CCW input fields can no longer be treated as sampling functions traveling at a fixed speed $v_{\text {NLE }}$. Instead, the speed of each sampling function changes as it propagates through the NLE because of the changing index. In spite of the added complexity the qualitative effect of finite NLE length is the same for phase-modulated operation as it is for amplitude-modulated operation.

Let us consider a specific example of the finitelength effect for amplitude-modulated operation. In this example the control pulse induces a steplike change in the attenuation of the NLE from $\alpha_{0}$ to $\alpha_{1}$. Therefore

$$
\begin{aligned}
\alpha(x, t) & =\alpha\left(t-x / v_{\mathrm{NLE}}\right) \\
& =\left\{\begin{array}{ll}
\alpha_{0} & \left(t-x / v_{\mathrm{NLE}}<0,0<x<L\right) \\
\alpha_{1} & \left(t-x / v_{\mathrm{NLE}}>0,0<x<L\right)
\end{array} .\right.
\end{aligned}
$$

It is straightforward to evaluate Eqs. (16) and (17) for this simple case, then to substitute $\gamma_{\mathrm{CW}}(t)$ and $\gamma_{\mathrm{CCW}}(t)$ into Eqs. (4) and (5). In Fig. 3(a) we plot $S(t)$ for the case in which the NLE lies entirely to one side of the midpoint. Arbitrary time units are used, with $T_{\text {transit }}=1$ and $\Delta t=1.5$. The finite length of the NLE adds a tail of total length $2 T_{\text {transit }}$ to the trailing edge of the $2 \Delta t$-wide rectangular sampling window. Although the detailed shape of the tail depends on the exact form of $\alpha(x, t)$, in general the effect is the same for all NLE's with the same transit time. By changing the sign of $\Delta t$ and $v_{\mathrm{NLE}}$, we can show that, if the NLE lies on the other side of the midpoint, the extra region is added to the leading edge of the rectangular
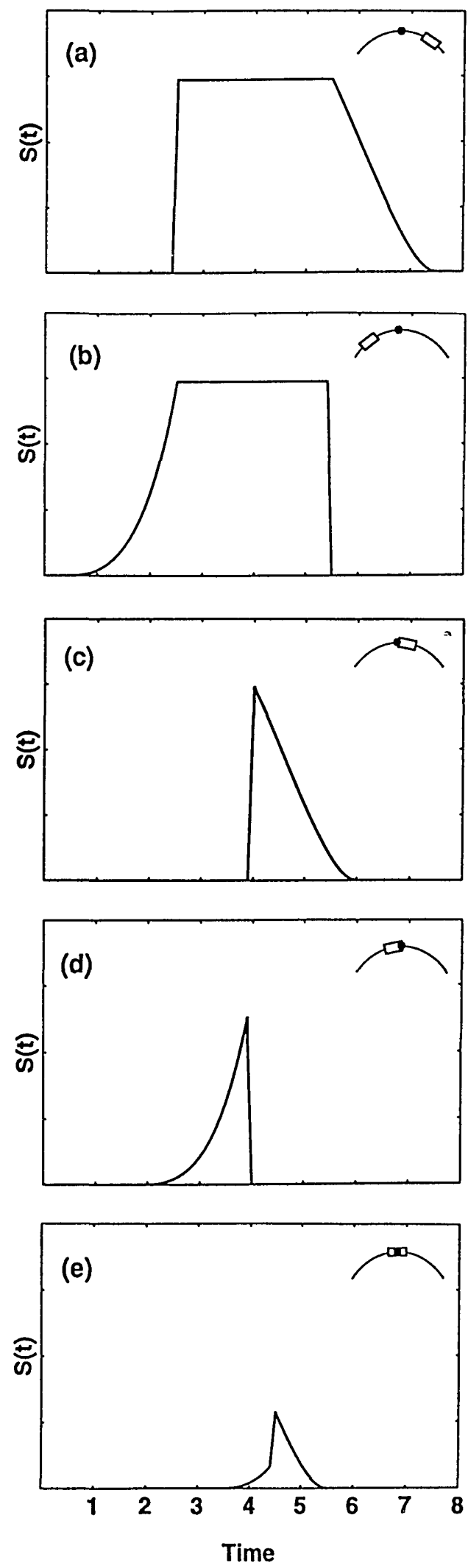

Fig. 3. Finite-length effect for different positions of the NLE. The sampling function $S(t)$ is plotted for the NLE position shown in the corner of each plot. (a), (b) The NLE is to the side of the midpoint; (c), (d) one edge of the NLE is at the midpoint; (e) the NLE is exactly centered in the loop. 
sampling window, as in Fig. 3(b). If the NLE is moved closer to the midpoint of the loop, $\Delta t$ can be reduced to zero as one edge of the NLE reaches the midpoint of the loop. Then all that remains is the extra region with a total length of $2 T_{\text {transit }}$ and a FWHM of approximately $T_{\text {transit }}$, as in Figs. 3(c) and (d). Centering the NLE even more closely in the loop does not reduce the duration of the sampling window. However, it does provide more symmetry, as shown in Fig. 3(e), in which the NLE is exactly centered in the loop. The improved symmetry of the sampling window is obtained at the expense of a lower peak value; compare Fig. 3(e) with Figs. 3(a)-3(d), which are plotted on the same vertical scale. In spite of the fact that the NLE is perfectly centered in the loop, it is nevertheless the asymmetry of the loop that causes the sampling window to exist. The asymmetry is expressed by the fact that the intraloop coupler appears on one side of the NLE rather than the other, as shown in Fig. 2.

If the length of the NLE is reduced, $T_{\text {transit }}$ can be reduced and a shorter sampling window can be obtained. However, the height of the sampling function is then reduced. Since the required optical output power of the switch is determined by subsequent processing stages, the power of the control pulse must be increased to restore the loss introduced by shortening the NLE. As a result, shortening the NLE does not permit any fundamental improvement in the gain-bandwidth product achievable from a given optical nonlinearity.

In the foregoing analysis we have disregarded the effect of the NLE's group-velocity dispersion. This is a legitimate approximation because the finitelength effect that we have described limits the short- est possible sampling window to approximately $T_{\text {transit }}=L n / c$, where $n$ is the refractive index, but the temporal broadening that is due to group-velocity dispersion limits the shortest sampling window to $\left(L \lambda_{0} \Delta \lambda / c\right) \mathrm{d}^{2} n / \mathrm{d} \lambda^{2}$, where $\lambda_{0}$ and $\Delta \lambda$ are the wavelength and the spectral width of the control signal, respectively. The first quantity is much larger than the second, and the finite-length effect therefore imposes a much stronger limitation than the groupvelocity dispersion effect.

\section{Experimental Results and Discussion}

In the case of a loop with large asymmetry our analysis shows that each control pulse causes two nonoverlapping sampling windows to be opened between input and output, identical but separated in time by $2 \Delta t$. To illustrate this result, we constructed a fiber loop mirror with the effect of the NLE simulated by a $\mathrm{LiNbO}_{3}$ electro-optic phase modulator, which is placed in the loop with a time asymmetry $\Delta t=360 \mathrm{~ns}$. (In order to accommodate this much asymmetry, the loop is $\sim 75 \mathrm{~m}$ long.) Using this electrically controlled element, we can simulate the behavior of a phase-modulated loop mirror for an arbitrary time-varying phase shift $\phi(t)$ by using a waveform generator to produce the electrical input to the phase modulator. In this experiment the electrical input is a 400-ns long ramp with its amplitude adjusted for $180^{\circ}$ peak-to-peak phase modulation, shown in Fig. 4(a). Since $2 \Delta t>T_{\text {control }}$, the condition for large asymmetry is satisfied. Although the generator and the phase modulator have relatively low bandwidth, we can simulate ultrafast operation by time scaling the time asymmetry $\Delta t$, the phase shift $\phi(t)$, and the sampling function $S(t)$ by the same

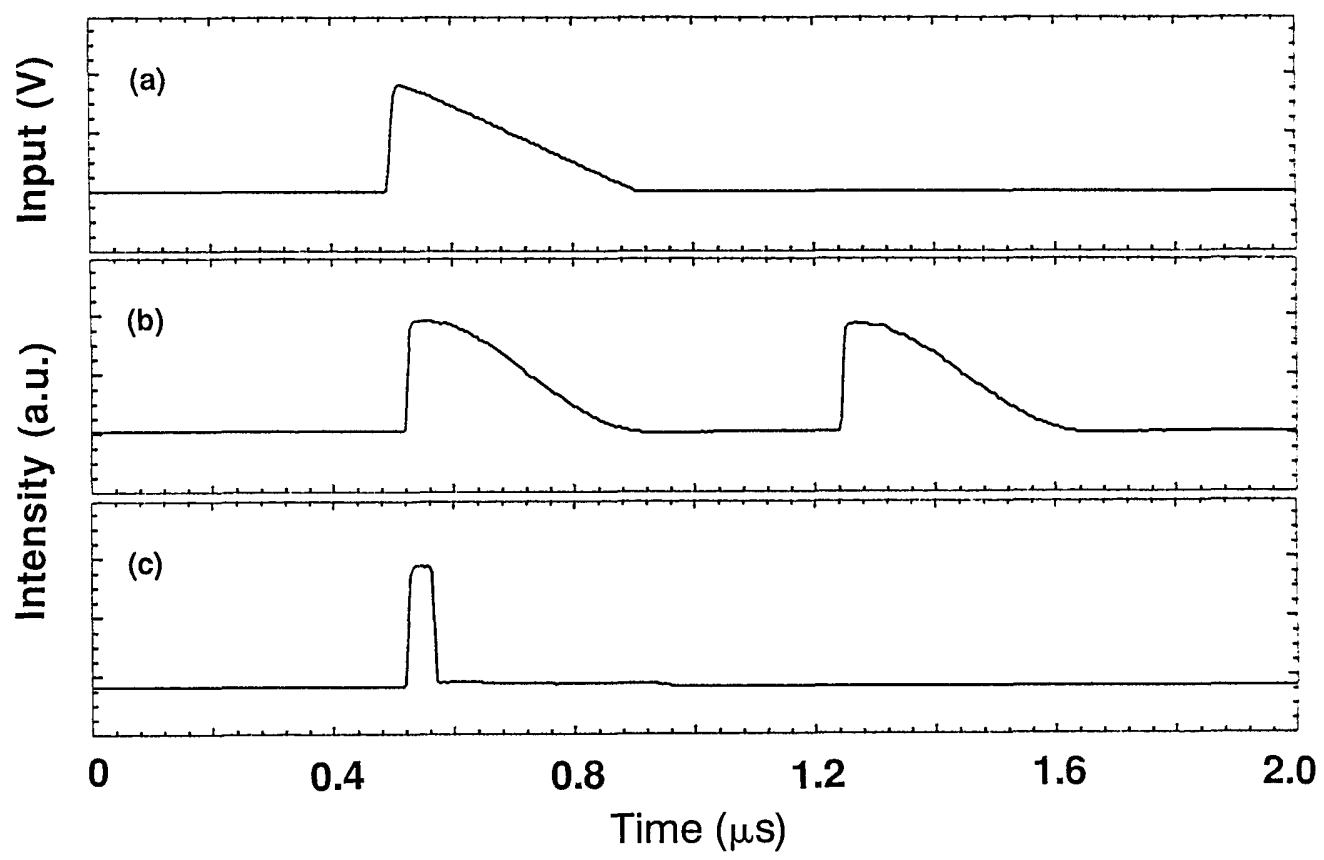

Fig. 4. Experimental data obtained with an electro-optic phase modulator used to simulate the effect of the nonlinear element. (a) The 400-ns ramp applied to the phase modulator. The output intensity is shown for two different positions of the phase modulator relative to the loop's midpoint. They correspond to a time asymmetry of (b) $360 \mathrm{~ns}$ (large asymmetry loop) and (c) $22 \mathrm{~ns}$ (small asymmetry loop). 
factor. A continous-wave optical signal from a 1320 -nm semiconductor laser is introduced into the loop's input so that the output intensity represents the sampling function $S(t)$. Figure 4(b) shows that two identical pulses emerge from the loop, separated by $2 \Delta t$. By simplifying Eq. (11) for the case of phase-modulated operation, we obtain an expression for the predicted sampling function:

$$
S(t)=A_{0}^{2}\left[\sin ^{2} \frac{\tilde{\phi}(t+\Delta t)}{2}+\sin ^{2} \frac{\tilde{\phi}(t-\Delta t)}{2}\right] .
$$

The phase shift induced by the phase modulator depends linearly on voltage, and therefore $\phi(t)$ is a linear function of time during the control pulse. Thus the intensity of each output pulse has a $\sin ^{2}(t)$ time dependence.

In the case of a loop with small asymmetry, our analysis shows that a short rectangular sampling window can be created from a single rapid transition, with the width of the window determined by the time asymmetry of the loop. To illustrate this result, we reduce the time asymmetry $\Delta t$ from $360 \mathrm{~ns}$ to $22 \mathrm{~ns}$. The $\mathrm{LiNbO}_{3}$ electro-optic phase modulator and the control pulse are the same as used for Fig. 4(b), but, because of the reduced asymmetry, the loop is now only a few meters long. The conditions for small asymmetry are satisfied: the rise time for the control pulse transitions is $10 \mathrm{~ns}$; thus $\tau_{\text {rise }}<\Delta t \ll \tau_{\text {fall }}$. Figure 4(c) shows the resulting sampling function $S(t)$. It consists of a rectangular pulse $2 \Delta t$ wide, generated by each control transition, in accordance with Eq. (12). After the pulse, a residual output intensity remains for the duration of the control pulse, caused by the finite fall time of the phase shift $\phi(t)$.

Now we cite our earlier results using a TOAD switch $^{13}$ to demonstrate the feasibility of all-optical switching in a loop with small asymmetry and to illustrate the finite-length effect. The configuration is the same as that of Fig. 3(a). The NLE is a $500-\mu \mathrm{m}$ InGaAsP polarization-insensitive travelingwave semiconductor optical amplifier with a transit time $T_{\text {transit }}$ of $\sim 5 \mathrm{ps}$. Optical pulses with a 2-ps FWHM are generated with a pulse-compressed $\mathrm{Nd}: \mathrm{YLF}$ laser, and they are split into fixed-delay, 20-fJ input pulses and variable-delay, 600-fJ control pulses. The input pulses and the control pulses are orthogonally polarized before they are introduced into the loop, and the control pulses are blocked at the output with a polarizer. The length of the loop in these experiments is $\sim 2 \mathrm{~m}$, but this length is irrelevant to the speed of the switch, which is controlled by the asymmetry of the NLE in the loop, which ranges from $10 \mathrm{~cm}$ to $1 \mathrm{~mm}$. The dominant optical nonlinearity arises from the gain saturation induced by the control pulse. The rise time $\tau_{\text {rise }}$ of the transmission modification is $\sim 1 \mathrm{ps},{ }^{22}$ but its fall time $\tau_{\text {fall }}$ and total duration $T_{\text {control }}$ are $\sim 800 \mathrm{ps}$. This pump-probe experiment actually measures the convolution of $S(t)$ with the input intensity $I_{\text {in }}(t)$. As a result, the measured sampling function has slower transitions than the true sampling function $S(t)$, and this effect is noticeable when the sampling function has a duration of the order of the 2-ps input pulse duration.

Figure 5(a) shows the measured sampling function for a time asymmetry $\Delta t$ of $345 \mathrm{ps}$. Since $\Delta t$ is neither much smaller nor much larger than the 800-ps recovery time of the NLE, this loop does not satisfy the conditions for large or small asymmetry. However, the zero-length approximation is valid, since $T_{\text {transit }} \ll \Delta t$. Equation (8) describes the sampling function under these conditions. Because the NLE is predominantly transmission modulated, the phase shift $\phi(t)$ can be treated as a constant, and Eq. (8) reduces to

$$
S(t)=1 / 4[A(t+\Delta t)-A(t-\Delta t)]^{2} .
$$

When the first term in brackets turns on, it yields the initial transient. Then the first term diminishes, and, after a time $2 \Delta t$ has elapsed, the second term turns on, creating a notch in the sampling function. Following this, both terms diminish with time, and the sampling function slowly returns to zero.

In Fig. 5(b) the time asymmetry $\Delta t$ is reduced to 65 ps, and therefore $\tau_{\text {rise }}<\Delta t \ll \tau_{\text {fall }}$, and this is a loop with small asymmetry. The zero-length approxima-

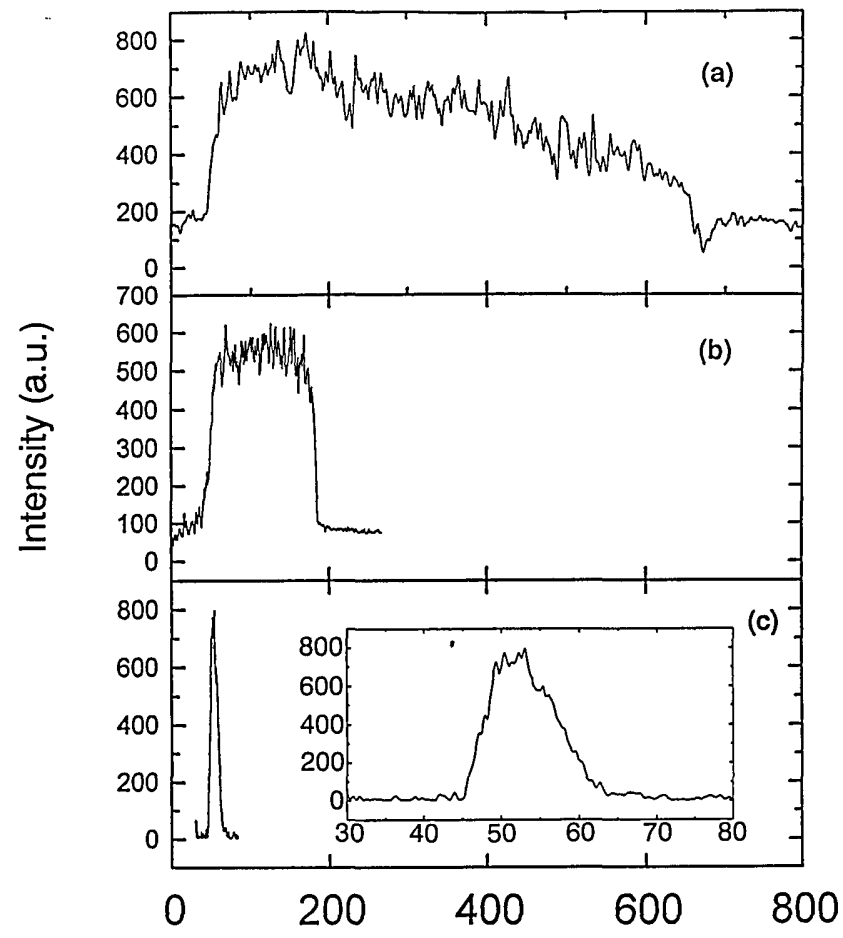

Time (ps)

Fig. 5. Results of an all-optical switching experiment obtained with a semiconductor optical amplifier used as a NLE. The pump-probe experiment yields a profile of the sampling window opened by the control pulse. The figure shows three different positions of the optical amplifier, corresponding to a time asymmetry of (a) $345 \mathrm{ps,} \mathrm{(b)} 65 \mathrm{ps,} \mathrm{and} \mathrm{(c)} 4 \mathrm{ps}$. 
tion is still valid, since $T_{\text {transit }} \ll \Delta t$. The sampling function is negligible outside a time window of duration $2 \Delta t$, and within the window it has a relatively constant level, given by Eq. (12). The rapid turn on of the gain saturation mechanism causes both the fast turn on and the fast turn off of the sampling function.

In Fig. 5(c) the time asymmetry $\Delta t$ is reduced to $\sim 4$ ps. This is also a small asymmetry loop, but in this case the zero-length approximation does not hold very well because $T_{\text {transit }} \approx \Delta t$. As a result, the sampling function shown in the inset detail is asymmetrical. Its leading edge has a rise time of a few picoseconds, partly because the measurement convolves the sampling function with the 2-ps long input pulse. But there is an extra region lasting $\sim 10 \mathrm{ps}$ attached to the trailing edge. The extra region arises from the finite-length effect, which adds an additional region $2 T_{\text {transit }}$ long to the trailing edge of the sampling function, as noted in Fig. 3(a). If subpicosecond control pulses were used and the NLE were centered more closely in the loop, the total duration of the sampling function would reach the minimum permitted by this NLE, which is $2 T_{\text {transit }}=10 \mathrm{ps,} \mathrm{with}$ a FWHM of $\sim 5 \mathrm{ps}$.

\section{Applications of the Small-Asymmetry Loop Mirror}

Our analysis has shown that, with sufficiently srnall asymmetry, a loop mirror with a nonlinear element in the loop can perform ultrafast switching. The time resolution of the switch is determined by the asymmetry of the NLE within the loop, not by the relaxation time of the nonlinearity. However, the relaxation time of the nonlinearity does limit the repetition rate at which switching can be performed. Therefore the applications for the switch are those that demand of a high sampling bandwidth but at a repetition rate that is much smaller than the bandwidth.

Two such applications are time-division optical demultiplexing and time sampling of repetitive optical signals. The usefulness of the small-asymmetry loop mirror for time-division demultiplexing has been demonstrated experimentally. ${ }^{14-16}$ For demultiplexing and sampling applications the duration of the sampling window must be short enough to sample the modulation bandwidth of the optical input effectively. However, these applications are not as demanding as general purpose ultrafast all-optical switching because they give the switch a long time to recover between sampling events. In the case of sampling repetitive signals the sampling can be performed at a low repetition rate, as is done frequently in electronic sampling. Similarly in the case of optical demultiplexing the switch does not need to route another optical pulse to the output until the next data frame, providing a recovery time as long as the data frame itself. If the small-asymmetry loop mirror is used in applications such as these, size and cost constraints demand that it be integrated on a substrate. Fortunately the size of the loop is completely irrelevant to switch operation as long as there is enough room in the loop for the required degree of asymmetry. As a result, the switch can easily be integrated, perhaps as part of a more complex optoelectronic integrated circuit.

\section{Conclusions}

In this paper we have described a general analytical framework for an optical loop mirror in which a nonlinear optical element is placed in the loop. We have shown that, when a NLE is placed in an optical loop mirror, two special types of loop exist, the small-asymmetry loop and the large-asymmetry loop. In a loop with small asymmetry a strong, slow nonlinearity can perform ultrafast all-optical switching in demultiplexing and sampling applications. Our analysis provides an analytical framework for the operation of the TOAD switch.

\section{References}

1. S. R. Friberg, A. M. Weiner, Y. Silberberg, B. G. Sfez, and P. S. Smith, "Femtosecond switching in a dual-core nonlinear coupler," Opt. Lett. 13, 904-906 (1988).

2. M. N. Islam, E. R. Sunderman, R. H. Stolen, W. Pleibel, and J. R. Simpson, "Soliton switching in a fiber nonlinear loop mirror," Opt. Lett. 14, 811-813 (1989).

3. M. W. Chbat, B. Hong, M. N. Islam, C. E. Soccolich, and P. R. Prucnal, "Ultrafast soliton-trapping AND gate," J. Lightwave Technol. 10, 2011-2016 (1992).

4. P. A. Andrekson, N. A. Olsson, J. R. Simpson, T. Tanbunek, R. A. Logan, and M. Haner, "16 Gbit/s all-optical demultiplexing using four-wave mixing," Electron. Lett. 27, 922-924 (1991).

5. A. Lattes, H. A. Haus, F. J. Leonberger, and E. P. Ippen, "An ultrafast all-optical gate," IEEE J. Quantum Electron. QE-19, 1718-1723 (1983).

6. S. G. Lee, P. A. Harten, J. P. Sokoloff, R. Jin, B. Fluegel, K. E. Meissner, C. L. Chuang, R. Binder, S. W. Koch, G. Khitrova, H. M. Gibbs, N. Peyghambarian, J. N. Polky, and G. A. Pubanz, "Femtosecond excitonic bleaching recovery in the optical Stark effect of $\mathrm{GaAs} / \mathrm{Al}_{x} \mathrm{Ga}_{1-x} \mathrm{As}$ multiple quantum wells and directional couplers," Phys. Rev. B 43, 1719-1725 (1991).

7. K. Al-Hemyari, J. S. Aitcheson, C. N. Ironside, G. T. Kennedy, R. S. Grant, and W. Sibbett, "Ultrafast all-optical switching in GaAlAs integrated interferometer in $1.55 \mu \mathrm{m}$ spectral region," Electron. Lett. 28, 1090-1092 (1992).

8. K. Otsuka, "Nonlinear antiresonant ring interferometer," Opt. Lett. 8, 471-473 (1983).

9. Y. Li, G. Eichmann, and R. R. Alfano, "Pulsed-mode laser Sagnac interferometry with applications in nonlinear optics and optical switching," Appl. Opt. 25, 209-214 (1986).

10. A. W. O'Neill and R. P. Webb, "All-optical loop mirror switch employing an asymmetric amplifier/attenuator combination," Electron. Lett. 26, 2008-2009 (1990).

11. M. C. Gabriel, N. A. Whitaker, C. W. Dirk, M. G. Kuzyk, and M. Thakur, "Measurement of ultrafast optical nonlinearities using a modified Sagnac interferometer," Opt. Lett. 16, 13341336 (1991).

12. M. Eiselt, "Optical loop mirror with semiconductor laser amplifier," Electron. Lett. 28, 1505-1507 (1992).

13. J. P. Sokoloff, P. R. Prucnal, I. Glesk, and M. Kane, "A terahertz optical asymmetric demultiplexer (TOAD)," in Photonics in Switching (Optical Society of America, Washington, D.C., 1993), paper PD4; IEEE Photon. Technol. Lett. 5, 787-790 (1993).

14. M. Eiselt, W. Pieper, and H. G. Weber, "All-optical demultiplex- 
ing with a semiconductor laser amplifier in a loop mirror configuration," Electron. Lett. 29, 1167-1168 (1993).

15. J. P. Sokoloff, I. Glesk, P. R. Prucnal, and R. K. Boncek, "Performance of a $50 \mathrm{Gbit} / \mathrm{s}$ optical time domain multiplexed system using a terahertz optical asymmetric demultiplexer," IEEE Photon. Technol. Lett. 6, 98-100 (1994).

16. I. Glesk, J. P. Sokoloff, and P. R. Prucnal, "Demonstration of all-optical demultiplexing of TDM data at $250 \mathrm{Gbit} / \mathrm{s}$," Electron. Lett. 30, 339-341 (1994).

17. E. J. Post, "Sagnac effect," Rev. Mod. Phys. 39, 475-494 (1967).

18. A. E. Siegman, "An antiresonant ring interferometer for coupled laser cavities, laser output coupling, mode locking, and cavity dumping," IEEE J. Quantum Electron. QE-9, 247-250 (1973).

19. D. B. Mortimore, "Fiber loop reflectors," J. Lightwave Technol. 6, 1217-1224 (1988).

20. T. A. Birks, "Jones calculus analysis of single-mode fiber Sagnac reflector," Appl. Opt. 27, 3107-3113 (1988).

21. M. P. Kesler and E. P. Ippen, "Subpicosecond gain dynamics in GaAlAs laser diodes," Appl. Phys. Lett. 51, 1765-1767 (1987).

22. J. Mark and J. Mork, "Subpicosecond gain dynamics in InGaAsP optical amplifiers: experiment and theory," Appl. Phys. Lett. 61, 2281-2283 (1992). 